\title{
Integrated Science General Education Program (ISGE): Bioastronomy Connections
}

\author{
Len Troncale \\ Biology 8 Institute for Advanced Systems Studies, California State \\ Polytechnic University, 3801 W. Temple Ave., Pomona, CA 91768, \\ U.S.A.
}

\begin{abstract}
A new, NSF-supported, General Education (GE) science curriculum, synthesizes and unifies the key theories and evidence of seven natural sciences using natural systems processes as Integrative Themes. The considerably reformulated subject matter is completely built on interdisciplinary concepts and methods fundamental to newly emerging crossdisciplinary fields like bioastronomy. The year of ISGE study incorporates 15 built-in computer based multimedia features and 10 special learning features to help non-science students learn more science, faster, and with better understanding. Results from seven test course offerings are reported. ISGE intends to be an initial example of the "living, evolving" knowledge bases needed for a space-faring species.
\end{abstract}

\section{Basic Idea}

Non-science students ( $90 \%$ of most university populations) must take at least four science courses (16 quarter/12 semester units) to fulfill their mandated general education. Often these students take four completely separated courses that have no reference to each other. The non-science student is left with unconnected, foreign information bits, isolated from each other, and more critically from student lives. In our ISGE (Integrated Science General Education Program), non-science students experience seven natural sciences, NOT as separate disciplines, but as different manifestations of the same mechanisms that are common to all "systems". Whatever the size of natural object studied, whether as tiny as subatomic particles $\left(10^{-14}\right)$, or a DNA interacting with protein $\left(10^{-8}\right)$, or a cell $\left(10^{-5}\right)$, or as large as a galaxy $\left(10^{20}\right)$, whether it is a natural or artificial system (CPU chip), all stable systems work by the same systems processes. These similarities are fundamental to their existence and behaviors.

\section{System Mechanisms as Integrative Themes}

Many different natural systems show forms that are hierarchical. ISGE teaches students what to look for to see this form in new cases and compares its function across all 7 sciences. Students learn the specifics of each new hierarchy overlayed on the common "learning" framework. This speeds learning and helps unify different phenomena. Besides hierarchies, ISGE uses fractals, cycles and cycling, 
feedback's, and other systems processes to explain diverse phenomena across the 7 sciences. Students first learn the key features and functions for a unifying theme (e.g. what is common to all "cycles and cycling"). Once understood, these make it faster and easier for students to absorb the specifics of stages found in star life cycles, cellular life cycles, the cycles of diverse oscillations, organism life cycles, and many more. By using these key "process" frameworks, students learn many things by learning one thing. The many similarities between distinct things at very different size scales help students understand unity.

\section{ISGE Attempts to Answer G.E. Problems Using Course Organi- zation}

ISGE began with a systems analysis of 20 obstacles inhibiting effective Science GE. Key ISGE features were designed to overcome several of these obstacles at the same time leading to a synergy of improvements mediated by the numerous ISGE features described below. For example, ISGE e-Games solve several problems simultaneously. Every week students use home computers to study at least 2 multimedia lessons. During the year of study, ISGE covers ALL of the science GE requirement. It uses a "hybrid" method that balances technology-based distanced learning with face-to-face, team-based discovery learning every week. Across a 3-week period, students focus on just one systems integrative theme, e.g. feedback mechanisms, learning specific cases in each of the 7 sciences. By years end they have studied a dozen systems integrative themes, and hundreds of case studies.

\section{ISGE Multimedia Enhances Learning}

Stunning Graphics Aid Learning: Humans are evolutionarily pre-adapted to absorb vast amounts of data through images. ISGE uses intriguing images from science that delight the senses and make use of this pre-adaptation to aid learning. Beautiful pictures focus the mind and command attention. These are important characteristics of a program designed to make science more appealing to non-science majors. Pictures are also superior at distilling key points and making them easy to remember as mental images. Brain research shows that long forgotten memories are still stored in our minds as images. Pictures also make complex ideas clear and simple in a human-oriented delivery format. ISGE makes serious use of the ancient aphorism, "A picture is worth a thousand words". Experimental results aimed at determining the comparative utility of conventional lectures versus computerized learning are summarized in the phrase, "tell me and I will learn 45\%; show me and I will learn 75\%". High Degree's of Interactivity Promote Discovery Learning: ISGE students choose among multiple alternative pathways to complete modules; these are not just page turning actions. They feel more engaged when actively following their own curiosity to discover knowledge. Many screens offer students dozens of freechoice buttons. Fourteen published higher education experiments consistently showed that interactive multimedia was much more effective than passive. The "direct" experience of interaction yields closer to $100 \%$ retention. Electronic "Games" Increase Learning: Many studies show students need more study time. 
Students love to play electronic games. ISGE modules have numerous, embedded games or experiences that cause them to "play" with the topic. The e-games extend study time. The "repetitions with endless variety" in e-games enhance understanding and increase problem-solving skills. All games are randomly generated. To beat them students must really know the material, and they learn it while trying to beat the game. Animations and Fly-Through's Deliver Understanding: Many science phenomena are too fast or slow for humans to see. Animations allow students to explore hidden dynamic processes. Fly-Throughs of cells, stars, atoms, planets, brains, controlled by students, increase interactivity and allow them to pick what to see. Ten Additional Multimedia Features Help Learning: ISGE systematically breaks down complex topics into their parts using "Stepwise Refinement". When students master a gross structure, finer steps of detail are sequentially added. In this way, non-science students gradually understand multileveled structures like chromosomes and muscles. "Electronic Glossaries" allow students to seek help for technical highlighted hot words when they encounter them in context. Students are presented with more than key definitions; they see graphics, significance, and links when they invoke each word in the e-glossary. ISGE includes many more multimedia features, e.g. "ISIS Gateways" (Integrated Science Internet Selections), and "Virtual Interviews".

\section{ISGE Interation Enhances Learning}

In ISGE, all natural science knowledge is presented as variant manifestations of one of a dozen system's mechanisms. They are the principal of several integrating strategies, each represented by an icon to alert students to the integration. Links connect different information in different modules on the same topic (e.g. DNA, or plate tectonics, are covered in several modules). Connections highlight fundamental similarities between any two sciences (e.g. the hierarchical structure of both DNA and computer codes). Bridges point to similarities between science and human phenomena (e.g. similar function of coins and ATP in similar networks of economics and metabolism). The Scientific Method is presented in 12 modules as an Integrative Theme. These relate science to student's daily lives for better motivation and show the world is one. The resulting ISGE Electronic e-Knowledge Base of the entire year of topics is available for direct student exploration. Transdisciplinary Labs provide experiences in 3 to 5 different science disciplines at one time, e.g. students explore hierarchical structure across 12 levels, from objects 2.0 to $10^{-18}$ meters in size, fully 20 orders of magnitude, from the human body to sub-atomic particles. All this in one lab. Labs are done in teams and require measurements, analysis, and graphing. The latest results of the Cognitive Sciences are included both as topics, and as part of the pedagogical design for integration. Students learn about neurons, neural nets, what impedes and helps learning, and the importance of "unlearning".

\section{ISGE Emphasizes Numerous Case Studies of Science Phenomena}

A main criticism of interdisciplinary courseware is lack of depth. A main criticism of American Science GE is weakness on detail. ISGE solves these serious problems by including $>250$ detailed case studies across the year of study. Each 
case study is a specific example of a key phenomenon of an Integrative Theme in one science. ISGE gives students summary, at-a-glance diagrams or mappings of the entire set of case studies. Each case study icon and/or is clickable (invokable; capable of being called up). Students can study them in different groupings based on pedagogy or interest. For, example, they can choose to study all integrative theme topics covered for any one discipline, e.g. the 31 topics covered in "astronomy". Or they can choose to study all specific phenomena covered for one Integrative Theme across the 6 sciences, e.g. 27 case studies of "feedback" across the several sciences. Students will be able to choose case studies using a unique "command cube" instead of the conventional pull-down lists. They can select any combo of themes and disciplines to study. So can the faculty.

\section{Testing ISGE: Demographics \& Student Performance}

A 3-month version of ISGE was offered to $>200$ students, in nine course offerings on 3 CSU campuses. These test/pilot courses were generally to Liberal Studies students, who are often "science phobic" and think they cannot learn science. Students were $76 \%$ female, $54 \%$ minority, $28 \%$ ESL, and a third below the age of 20. Most were teacher preparation students to obtain a "multiplier effect". This science-challenged population was tested for short and long-term learning using 900 points of quizzes, multiple choice \& essay exams, homework, and labs. Both long and short-term memory were tested, and both individual and team products and homework were scored. Reproducible results were obtained. $75 \%$ of the population consistently earned A's (90\% of challenges fulfilled) and B's $(80 \%)$. These are very solid results for an audience that dislikes science.

\section{Do Students Like ISGE?}

Students were asked to judge 111 ISGE Features by choosing whether that feature was better than conventional education or not based on a Likert scale of 1 to 7,7 being best. 3 to 1 were "poor to worst", 4 = "same as", and 5-7 were "better", "much better", and "very much better". The grand mode for the entire sample was a score of "6" meaning most ISGE Features were judged "much better than" conventional courses. Students judged 31 of the 101 Features "very much better than" the conventional courseware. Students concluded that $99 \%$ of ISGE Features were better than those encountered in conventional college courses. These are the same multimedia and learning features described above. Pre- and post-course attitudes to science were also measured. Post-course attitudes toward science after ISGE improved significantly for 12 measures, but declined for 5 measures. All ten categories of ISGE learning methods were rated as better than conventional courses.

\section{Conclusion and ISGE Relation to BioAstronomy}

ISGE delivers a rigorous training in skills and concepts basic to cross-disciplinary fields like bioastronomy and systems biology. It could, therefore, be used as a portion of such educational programs, or as an outreach program delivered 
to the general education academic population to attract some to these new specialties. ISGE also includes many modules of direct interest to bioastronomy on Origins of the Universe, Origins of Stars, Origins of Solar Systems, Origins of Brown Matter, and Origins of Life. It has modules on key astronomical tools and evidence, such as Red Shifts, Herzprung-Russell Diagrams, key telescope systems, particle accelerators, etc. It has case studies on Dark Matter, Dark Energy, and other current mysteries. The highly interconnected ISGE knowledge base and focus on systems processes give students the whole system experience needed for those who will encounter totally new natural systems manifestations in space travel. They will have to be very quick studies in new encounters; they will need the overview provided by general systems approaches, as well as the detail learned from numerous case studies. They will be aided by trained skills as seeing the large in the small, and the small in the large gained from experiences like the ISGE Program. 